DOI: $10.15393 / \mathrm{j} 3 . \operatorname{art} .2020 .6750$

UDC 517.98

Mohammed Shehu Shagari, Akbar Azam

\title{
FIXED POINT THEOREMS OF FUZZY SET-VALUED MAPS WITH APPLICATIONS
}

\begin{abstract}
In this paper, we introduce the notion of Suzuki-type $(\alpha, \beta)$-weak contractions in the setting of fuzzy set-valued maps, thereby establishing some fuzzy fixed point theorems. Moreover, one of our theorems is applied to study a homotopy result.
\end{abstract}

Key words: Fuzzy set, Fuzzy set-valued mapping, Fixed point, Weak contraction

2010 Mathematical Subject Classification: 46S40, 47H10, 54H25, 34A12.

1. Introduction. Fixed-point theory is one of the most active research fields in modern nonlinear functional analysis. A typical fixedpoint equation is of the form $T x=x$, where $T$ is a self-mapping on a non-empty set $X$. This problem can be reformulated as $g(x)=0$, where $g(x)=x-T x$. Though the problem statement is simple, finding a solution may be extremely difficult, and sometimes not possible. The earliest affirmative response to this problem was announced by Banach [4] under some suitable conditions: when $T$ is a contraction and $X$ is endowed with a norm such that the corresponding topology yields completeness. In 1969, Kannan [13] gave an analogue sort of contractive condition that demonstrated the existence of a fixed point. The basic distinction between the Banach fixed-point theorem (BFT) and that of the Kannan contraction is that continuity of contraction is not needed in the later. Analogous well-known generalizations of the BFT were established by Chatterjea [7] and Edelstein [10]. In the last six decades, the above results have been extended in different directions. Along the way, the notion of weak contraction was introduced by Berinde [5]. The idea generalized the well-celebrated fixed-point theorems due to Banach [4], Chatterjea [7], Zamfirescu [22], and many others. It is well-known that the Banach contraction theorem cannot characterize metric completeness. To resolve this

(C) Petrozavodsk State University, 2020 
problem, a generalization of the BFT was given by Suzuki [19]. Later on, an interesting improvement of the Suzuki fixed-point theorem in the setting of multivalued mappings was presented by Djoric and Lazovic [9].

Along the lane, the area of applied mathematics witnessed tremendous developments as a result of the introduction of fuzzy sets by Zadeh [21]. Classically, fuzzy set is characterized by a membership function that assigns to each element a grade of membership between zero and one. Later, Weiss [20] and Butnairu [6] initiated the study of fixed points of fuzzy mappings. Whereas fixed point theorems for fuzzy set-valued mappings have been investigated by Heilpern [11], who originated the idea of fuzzy contractions and proved a fixed-point theorem parallel to the BanachCacciopoli principle in the frame of fuzzy sets. Thereafter, several authors have studied and applied fuzzy fixed-point results in different directions; see, for example, [1-3], [12], [14-18], and the references therein.

In this work, motivated by the ideas of Suzuki [19], Djoric and Lazovic [9], we define the notion of Suzuki-type $(\alpha, \beta)$-weak contractions in the setting of fuzzy set-valued maps. As a result, fuzzy fixed-point theorem of Suzuki-type is proved and some consequences are obtained thereafter. In addition, an application in homotopy result is established to highlight the usability of one of our results.

2. Fuzzy and Multivalued Mappings. In this section, we present some useful definitions and basic concepts from the literature, which will be needed in the sequel. Let $(X, d)$ be a metric space. We denote by $C B(X)$ the class of all nonempty, closed, and bounded subsets of $X$. Let $H(\cdot, \cdot)$ be the Hausdorff metric on $C B(X)$ induced by $d$, that is,

$$
H(A, B)=\max \left\{\sup _{a \in A} d(a, B), \sup _{b \in B} d(A, b)\right\}
$$

for $A, B \in C B(X)$, where $d(x, A)=\inf \{d(x, a): a \in A\}$. A point $u$ in $X$ is a fixed point of a multi-valued mapping $T: X \longrightarrow C B(X)$ if $u \in T u$. Recall that an ordinary subset $A$ of $X$ is determined by its characteristic function $\chi_{A}$, defined by $\chi_{A}: A \rightarrow\{0,1\}$ :

$$
\chi_{A}(x)= \begin{cases}1, & \text { if } x \in A \\ 0, & \text { if } x \notin A .\end{cases}
$$

The value $\chi_{A}(x)$ specifies whether an element belongs to $A$ or not. This idea is used to define fuzzy sets by allowing an element $x \in A$ to assume 
any possible value of the interval $[0,1]$. Thus, a fuzzy set $A$ in $X$ is a set of ordered pairs given as

$$
A=\left\{\left(x, \mu_{A}(x)\right): x \in X\right\},
$$

where $\mu_{A}: X \rightarrow[0,1]=I$ and $\mu_{A}(x)$ is called the membership function of $x$, or the degree to which $x \in X$ belongs to the fuzzy set $A$.

Throughout this paper, we shall let $(0,1]=I^{+1}$ and $(0,1)=I_{-1}$. An important notion in fuzzy set theory is that of an $\alpha$-level set. If $A$ is a fuzzy set in $X$, the (crisp) set of elements in $X$ belonging to $A$ at least of degree $\alpha \in I^{+1}$ is called the $\alpha$-level set, denoted by $[A]_{\alpha}$. That is,

$$
[A]_{\alpha}=\left\{x \in X: \mu_{A}(x) \geq \alpha\right\}
$$

On the other hand,

$$
[A]_{\alpha}^{*}=\left\{x \in X: \mu_{A}(x)>\alpha\right\}
$$

is called the strong $\alpha$-level set or strong $\alpha$-level cut. Denote by $I^{X}$ the family of all fuzzy sets in $X$. Let $X$ be an arbitrary set and $Y$ be a metric space. A mapping $T: X \rightarrow I^{Y}$ is called fuzzy mapping. A fuzzy mapping $T$ is a fuzzy subset of $X \times Y$. The function $T(x)(y)$ is the membership value of $y$ in $T(x)$. An element $u$ in $X$ is said to be a fuzzy fixed point of $T$ if there exists an $\alpha \in I^{+1}$ such that $u \in[T u]_{\alpha}$. Denote the set of all fixed points of $T$ by $\mathcal{F}_{i x}(T)$.

\section{Example 1.}

Let $X=[-5,5]$ and $Y=[-5,5]$. Define $T: X \rightarrow I^{Y}$ by

$$
T(x)(y)=\cos ^{2} x \cos ^{2} y
$$

for all $x \in X$ and $y \in Y$. Then $T$ is a fuzzy mapping. Graphical representation of the fuzzy mapping in Example 1 showing all possible membership values of $y$ in $T(x)$ is in Figure 1.

Throughout this paper, the function $r: I_{-1} \longrightarrow\left(\frac{1}{2}, 1\right]$ is defined by

$$
r(\alpha)= \begin{cases}1, & \text { if } 0<\alpha<\frac{1}{2} \\ 1-\alpha, & \text { if } \frac{1}{2} \leq \alpha<1\end{cases}
$$

For $x, y \in X$ and $\alpha \in I^{+1}$, define 


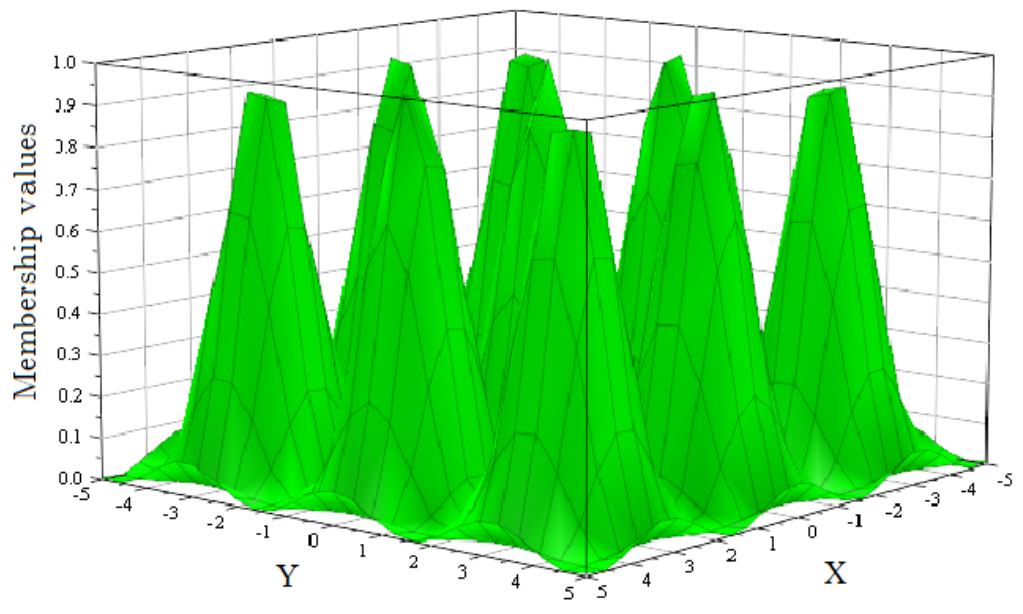

Figure 1: Graphical representation of the fuzzy mapping in Example 1

$$
\begin{aligned}
\bigvee(x, y)=\max \left\{d(x, y), d\left(x,[T x]_{\alpha}\right), d\left(y,[T y]_{\alpha}\right)\right. \\
\left.\frac{d\left(x,[T y]_{\alpha}\right)+d\left(y,[T x]_{\alpha}\right)}{2}, \frac{d\left(x,[T x]_{\alpha}\right) d\left(y,[T y]_{\alpha}\right)}{1+d(x, y)}\right\}
\end{aligned}
$$

and

$$
\bigwedge(x, y)=\min \left\{d\left(x,[T x]_{\alpha}\right), d\left(y,[T x]_{\alpha}\right), \frac{d\left(x,[T x]_{\alpha}\right) d\left(y,[T y]_{\alpha}\right)}{1+d(x, y)}\right\} .
$$

3. Fuzzy Fixed Points of Suzuki-type $(\alpha, \beta)$-Weak Contractions. In this section, we present a fuzzy fixed-point theorem of Suzukitype $(\alpha, \beta)$-weak contractions. First, we give the following definition.

Definition 1. A fuzzy mapping $T: X \rightarrow I^{X}$ is called a Suzuki-type $(\alpha, \beta)$-weak contraction, if for all $x, y \in X$ with $x \neq y$ there exist some $\alpha \in I_{-1}$ and $\beta \geq 0$, such that

$$
r(\alpha) d\left(x,[T x]_{\alpha}\right) \leq d(x, y)
$$

implies

$$
H\left([T x]_{\alpha},[T y]_{\alpha}\right) \leq \alpha \bigvee(x, y)+\beta \bigwedge(x, y)
$$


Theorem 1. Let $(X, d)$ be a complete metric space and $T: X \rightarrow I^{X}$ be a Suzuki-type $(\alpha, \beta)$-weak contraction. Assume that for each $x \in X$ there exists $\alpha \in I_{-1}$, such that $[T x]_{\alpha}$ is a non-empty closed and bounded subset of $X$. Then $\mathcal{F}_{i x}(T) \neq \emptyset$.

Proof. Let $\alpha_{1} \in I_{-1}$ be such that $0<\alpha \leq \alpha_{1}<1, x_{1} \in X$ and $\rho=\frac{1}{\sqrt{\alpha}}$. Then, by the assumption, $\left[T x_{1}\right]_{\alpha}$ is nonempty. Therefore, we can find $x_{2} \in X$ such that $x_{2} \in\left[T x_{1}\right]_{\alpha}$. Since $\rho>1$, choose $x_{3} \in\left[T x_{2}\right]_{\alpha}$, such that

$$
d\left(x_{2}, x_{3}\right) \leq \rho H\left(\left[T x_{1}\right]_{\alpha},\left[T x_{2}\right]_{\alpha}\right) .
$$

If $x_{1}=x_{2}$, then $x_{1} \in\left[T x_{1}\right]_{\alpha}$ for some $\alpha \in I_{-1}$, and the theorem is proved. Assume that $x_{1} \neq x_{2}$. Since $r(\alpha) \leq 1$,

$$
r(\alpha) d\left(x_{1},\left[T x_{1}\right]_{\alpha}\right) \leq d\left(x_{1},\left[T x_{1}\right]_{\alpha}\right) \leq d\left(x_{1}, x_{2}\right)
$$

implies

$$
\begin{aligned}
& d\left(x_{2}, x_{3}\right) \leq \rho H\left(\left[T x_{1}\right]_{\alpha},\left[T x_{2}\right]_{\alpha}\right) \frac{1}{\sqrt{\alpha}}(\alpha \bigvee(x, y)+\beta \bigwedge(x, y)) \leq \\
& \leq \sqrt{\alpha} \max \left\{d\left(x_{1}, x_{2}\right), d\left(x_{1},\left[T x_{1}\right]_{\alpha}\right), d\left(x_{2},\left[T x_{2}\right]_{\alpha}\right),\right. \\
& \left.\frac{d\left(x_{1},\left[T x_{2}\right]_{\alpha}\right)+d\left(x_{2},\left[T x_{1}\right]_{\alpha}\right)}{2}, \frac{d\left(x_{1},\left[T x_{1}\right]_{\alpha}\right) d\left(x_{2},\left[T x_{2}\right]_{\alpha}\right)}{1+d\left(x_{1}, x_{1}\right)}\right\}+ \\
& +\frac{\beta}{\sqrt{\alpha}} \min \left\{d\left(x_{1},\left[T x_{1}\right]_{\alpha}\right), d\left(x_{2},\left[T x_{1}\right]_{\alpha}\right), \frac{d\left(x_{1},\left[T x_{1}\right]_{\alpha}\right) d\left(x_{2},\left[T x_{2}\right]_{\alpha}\right)}{1+d\left(x_{1}, x_{2}\right)}\right\} \leq \\
& \leq \sqrt{\alpha} \max \left\{d\left(x_{1}, x_{2}\right), d\left(x_{2}, x_{3}\right), \frac{d\left(x_{1}, x_{3}\right)+d\left(x_{2}, x_{2}\right)}{2}, \frac{d\left(x_{1}, x_{2}\right) d\left(x_{2}, x_{3}\right)}{1+d\left(x_{1}, x_{2}\right)}\right\}+ \\
& +\frac{\beta}{\sqrt{\alpha}} \min \left\{d\left(x_{1}, x_{2}\right), d\left(x_{2}, x_{2}\right), \frac{d\left(x_{1}, x_{2}\right) d\left(x_{2}, x_{3}\right)}{1+d\left(x_{1}, x_{2}\right)}\right\} \leq \\
& \leq \sqrt{\alpha} \max \left\{d\left(x_{1}, x_{2}\right), d\left(x_{2}, x_{3}\right), \frac{d\left(x_{1}, x_{2}\right)+d\left(x_{2}, x_{3}\right)}{2}\right\} \leq \\
& \leq \sqrt{\alpha} \max \left\{d\left(x_{1}, x_{2}\right), d\left(x_{2}, x_{3}\right)\right\} .
\end{aligned}
$$

If $d\left(x_{1}, x_{2}\right) \leq d\left(x_{2}, x_{3}\right)$, then from (1) we have

$$
d\left(x_{2}, x_{3}\right) \leq \sqrt{\alpha} d\left(x_{2}, x_{3}\right)<d\left(x_{2}, x_{3}\right)
$$

a contradiction. Hence, $d\left(x_{1}, x_{2}\right)>d\left(x_{2}, x_{3}\right)$ and (1) becomes

$$
d\left(x_{2}, x_{3}\right) \leq \sqrt{\alpha} d\left(x_{1}, x_{2}\right) \leq \sqrt{\alpha_{1}} d\left(x_{1}, x_{2}\right) .
$$


Continuing in this way, we generate a sequence $\left\{x_{n}\right\}_{n \in \mathbb{N}}$ in $X$, such that $x_{n+1} \in\left[T x_{n}\right]_{\alpha}$ and

$$
d\left(x_{n}, x_{n+1}\right) \leq \sqrt{\alpha_{1}} d\left(x_{n-1}, x_{n}\right)
$$

from which we have

$$
\sum_{n=1}^{\infty} d\left(x_{n}, x_{n+1}\right) \leq \sum_{n=1}^{\infty}\left(\sqrt{\alpha_{1}}\right)^{n-1} d\left(x_{1}, x_{2}\right)<\infty .
$$

By standard argument, we conclude that $\left\{x_{n}\right\}_{n \in \mathbb{N}}$ is a Cauchy sequence in $X$. The completeness of $X$ implies that there exists $u \in X$, such that $x_{n} \rightarrow u$ as $n \rightarrow \infty$.

Claim: for all $u \neq z$, we have

$$
d\left(u,[T z]_{\alpha}\right) \leq \alpha \max \left\{d(u, z), d\left(z,[T z]_{\alpha}\right)\right\} .
$$

Since $x_{n} \rightarrow u$ as $n \rightarrow \infty$, there exists a positive integer $m$, such that

$$
d\left(x_{n}, u\right) \leq \frac{1}{3} d(u, z), \text { for all } n \geq m .
$$

Given that $x_{n+1} \in\left[T x_{n}\right]_{\alpha}$, we get

$$
\begin{aligned}
r(\alpha) d\left(x_{n},\left[T x_{n}\right]_{\alpha}\right) \leq d\left(x_{n},\left[T x_{n}\right]_{\alpha}\right) & \leq d\left(x_{n}, x_{n+1}\right) \leq \\
& \leq d\left(x_{n}, u\right)+d\left(u, x_{n+1}\right) \leq \frac{2}{3} d(u, z) .
\end{aligned}
$$

Thus, for $n \geq m$ we have

$$
\begin{aligned}
& r(\alpha) d\left(x_{n},\left[T x_{n}\right]_{\alpha}\right) \leq \frac{2}{3} d(u, z)=d(u, z)-\frac{1}{3} d(u, z) \leq \\
& \quad \leq d(u, z)-d\left(u, x_{n}\right) \leq d\left(x_{n}, z\right) .
\end{aligned}
$$

Hence,

$$
r(\alpha) d\left(x_{n},\left[T x_{n}\right]_{\alpha}\right) \leq d\left(x_{n}, z\right)
$$

implies

$$
\begin{aligned}
d\left(x_{n+1},[T z]_{\alpha}\right) & \leq H\left(\left[T x_{n}\right]_{\alpha},[T z]_{\alpha}\right) \leq \\
\leq & \alpha \max \left\{d\left(x_{n}, z\right), d\left(x_{n},\left[T x_{n}\right]_{\alpha}\right), d\left(z,[T z]_{\alpha}\right),\right.
\end{aligned}
$$




$$
\begin{gathered}
\left.\frac{d\left(x_{n},[T z]_{\alpha}\right)+d\left(z,\left[T x_{n}\right]_{\alpha}\right)}{2}, \frac{d\left(x_{n},\left[T x_{n}\right]_{\alpha}\right) d\left(z,[T z]_{\alpha}\right)}{1+d\left(x_{n}, z\right)}\right\}+ \\
+\beta \min \left\{d\left(x_{n},\left[T x_{n}\right]_{\alpha}\right), d\left(z,\left[T x_{n}\right]_{\alpha}\right), \frac{d\left(x_{n},\left[T x_{n}\right]_{\alpha}\right) d\left(z,[T z]_{\alpha}\right)}{1+d\left(x_{n}, z\right)}\right\} .
\end{gathered}
$$

From (5), we have

$$
\begin{aligned}
& d\left(x_{n+1},[T z]_{\alpha}\right) \leq \alpha \max \left\{d\left(x_{n}, z\right), d\left(x_{n}, x_{n+1}\right), d\left(z,[T z]_{\alpha}\right),\right. \\
&\left.\frac{d\left(x_{n}[T z]_{\alpha}\right)+d\left(z, x_{n+1}\right)}{2}, \frac{d\left(x_{n},\left[T x_{n}\right]_{\alpha}\right) d\left(z,[T z]_{\alpha}\right)}{1+d\left(x_{n}, z\right)}\right\}+ \\
&+\beta \min \left\{d\left(x_{n}, x_{n+1}\right), d\left(z, x_{n+1}\right), \frac{d\left(x_{n}, x_{n+1}\right) d\left(z,[T z]_{\alpha}\right)}{1+d\left(x_{n}, z\right)}\right\} .
\end{aligned}
$$

As $n \rightarrow \infty$ in (6), we obtain

$$
\begin{aligned}
& d\left(u,[T z]_{\alpha}\right) \leq \alpha \max \left\{d(u, z), d\left(z,[T z]_{\alpha}\right), \frac{d\left(u,[T z]_{\alpha}\right)+d(u, z)}{2}\right\}+ \\
&+\beta \min \{0, d(u, z)\} \leq \alpha \max \left\{d(u, z), d\left(z,[T z]_{\alpha}\right)\right\}
\end{aligned}
$$

Now, to show that $u \in[T u]_{\alpha}$ for some $\alpha \in I_{-1}$, we consider the following two possible cases:

Case(i): $0<\alpha<\frac{1}{2}$.

Suppose that for all $\alpha \in I_{-1}, u \neq p, u \notin[T u]_{\alpha}$ and $p \in[T u]_{\alpha}$ such that $d(p, u)<d\left(u,[T u]_{\alpha}\right)$. Setting $z=p$ in (2), we have

$$
d\left(u,[T p]_{\alpha}\right) \leq \alpha \max \left\{d(u, p), d\left(p,[T p]_{\alpha}\right)\right\} .
$$

Now,

$$
r(\alpha) d\left(u,[T u]_{\alpha}\right) \leq d\left(u,[T u]_{\alpha}\right) \leq d(u, p)
$$

implies

$$
\begin{gathered}
d\left(p,[T p]_{\alpha}\right) \leq H\left([T u]_{\alpha},[T p]_{\alpha}\right) \leq \alpha \max \left\{d(u, p), d\left(u,[T u]_{\alpha}\right), d\left(p,[T p]_{\alpha}\right),\right. \\
\left.\frac{d\left(u,[T p]_{\alpha}\right)+d\left(p,[T u]_{\alpha}\right)}{2}, \frac{d\left(u,[T u]_{\alpha}\right) d\left(p,[T p]_{\alpha}\right)}{1+d(u, p)}\right\}+ \\
+\beta \min \left\{d\left(u,[T u]_{\alpha}\right), d\left(p,[T u]_{\alpha}\right), \frac{d\left(u,[T u]_{\alpha}\right) d\left(p,[T p]_{\alpha}\right)}{1+d(u, p)}\right\} \leq
\end{gathered}
$$




$$
\begin{gathered}
\leq \alpha \max \left\{d(u, p), d\left(p,[T p]_{\alpha}\right), \frac{d\left(u,[T p]_{\alpha}\right)}{2}, \frac{d(u, p) d\left(p,[T p]_{\alpha}\right)}{1+d(u, p)}\right\}+ \\
+\beta \min \left\{d(u, p), 0, \frac{d(u, p) d\left(p,[T p]_{\alpha}\right)}{1+d(u, p)}\right\} \leq \\
\leq \alpha \max \left\{d(u, p), d\left(p,[T p]_{\alpha}\right), \frac{d(u, p)+d\left(p,[T p]_{\alpha}\right)}{2}\right\} \leq \\
\leq \alpha \max \left\{d(u, p), d\left(p,[T p]_{\alpha}\right)\right\} .
\end{gathered}
$$

Assume that $d(u, p) \leq d\left(p,[T p]_{\alpha}\right)$; then from (8) we have

$$
d\left(p,[T p]_{\alpha}\right) \leq \alpha d\left(p,[T p]_{\alpha}\right)<d\left(p,[T p]_{\alpha}\right):
$$

a contradiction. Hence, $d(u, p)>d\left(p,[T p]_{\alpha}\right)$, and

$$
d\left(p,[T p]_{\alpha}\right) \leq \alpha d(u, p)<d(u, p)
$$

Therefore, (7) becomes $d\left(u,[T p]_{\alpha}\right) \leq \alpha d(u, p)$. Consequently,

$$
\begin{aligned}
& d\left(u,[T u]_{\alpha}\right) \leq d\left(u,[T p]_{\alpha}\right)+H\left([T p]_{\alpha},[T u]_{\alpha}\right) \leq \\
& \leq d\left(u,[T p]_{\alpha}\right)+\alpha \max \left\{d(u, p), d\left(p,[T p]_{\alpha}\right)\right\} \leq \alpha d(u, p)+\alpha d(u, p)= \\
& =2 \alpha d(u, p)<d(u, p)<d\left(u,[T u]_{\alpha}\right), \text { (in view of assumption) }
\end{aligned}
$$

yields a contradiction. Therefore, $u \in[T u]_{\alpha}$ for some $\alpha \in I_{-1}$.

Case(ii): $\frac{1}{2} \leq \alpha<1$.

For this, we shall prove that

$$
\begin{aligned}
& H\left([T z]_{\alpha},[T u]_{\alpha}\right) \leq \alpha \max \left\{d(u, z), d\left(z,[T z]_{\alpha}\right), d\left(u,[T u]_{\alpha}\right),\right. \\
&\left.\frac{d\left(z,[T u]_{\alpha}\right)+d\left(u,[T z]_{\alpha}\right)}{2}, \frac{d\left(z,[T z]_{\alpha}\right) d\left(u,[T u]_{\alpha}\right)}{1+d(u, z)}\right\}+ \\
&+ \beta \min \left\{d\left(z,[T z]_{\alpha}\right), d\left(u,[T z]_{\alpha}\right), \frac{d\left(u,[T u]_{\alpha}\right) d\left(z,[T z]_{\alpha}\right)}{1+d(u, z)}\right\},
\end{aligned}
$$

holds for all $z \in X$ with $z \neq u$. Now, for each $m \in \mathbb{N}$, there exists $v_{m} \in[T z]_{\alpha}$, such that

$$
d\left(u, v_{m}\right) \leq d\left(u,[T z]_{\alpha}\right)+\frac{1}{5 m} d(u, z) .
$$

Thus, we have 


$$
\begin{aligned}
d\left(z,[T z]_{\alpha}\right) \leq d\left(z, v_{m}\right) \leq d(z, u)+ & d\left(u, v_{m}\right) \leq \\
& \leq d(z, u)+d\left(u,[T z]_{\alpha}\right)+\frac{1}{5 m} d(z, u) .
\end{aligned}
$$

Using (2), we have

$$
d\left(z,[T z]_{\alpha}\right) \leq d(u, z)+\alpha \max \left\{d(u, z), d\left(z,[T z]_{\alpha}\right)\right\}+\frac{1}{5 m} .
$$

If $d(z, u)>d\left(z,[T z]_{\alpha}\right)$, then from (11) we get

$$
d\left(z,[T z]_{\alpha}\right) \leq d(u, z)+\alpha d(u, z)+\frac{1}{5 m} d(u, z)=\left[(1+\alpha)+\frac{1}{5 m}\right] d(u, z),
$$

from which we have

$$
\left[\frac{1}{1+\alpha}\right] d\left(z,[T z]_{\alpha}\right) \leq\left[1+\frac{1}{5(1+\alpha) m}\right] d(u, z) .
$$

Using $r(\alpha)=1-\alpha$, we get

$$
\begin{aligned}
r(\alpha) d\left(z,[T z]_{\alpha}\right)=(1-\alpha) d\left(z,[T z]_{\alpha}\right) \leq & \left(\frac{1}{1+\alpha}\right) d\left(z,[T z]_{\alpha}\right) \leq \\
& \leq\left[1+\frac{1}{5(1+\alpha) m}\right] d(u, z) .
\end{aligned}
$$

As $m \rightarrow \infty$ in (12), we have

$$
r(\alpha) d\left(z,[T z]_{\alpha}\right) \leq d(u, z) .
$$

On the other hand, if $d(u, z)<d\left(z,[T z]_{\alpha}\right)$, then (11) gives

$$
d\left(z,[T z]_{\alpha}\right) \leq d(u, z)+\alpha d\left(z,[T z]_{\alpha}\right)+\frac{1}{5 m} d(u, z),
$$

which yields

$$
(1-\alpha) d\left(z,[T z]_{\alpha}\right) \leq\left(1+\frac{1}{5 m}\right) d(u, z)
$$

As $m \rightarrow \infty$ in (13), we have

$$
(1-\alpha) d\left(z,[T z]_{\alpha}\right) \leq d(u, z) .
$$

This shows that $r(\alpha) d\left(z,[T z]_{\alpha}\right) \leq d(u, z)$, which, by Definition 1 , implies (10). Moreover, since $x_{n+1} \neq x_{n}$ for all $n \in \mathbb{N}$, then $u \neq x_{n+1}$. Therefore, setting $x_{n}=z$ in (10), we have 


$$
\begin{gathered}
d\left(x_{n+1},[T u]_{\alpha}\right) \leq H\left(\left[T x_{n}\right]_{\alpha},[T u]_{\alpha}\right) \leq \\
\leq \alpha \max \left\{d\left(x_{n}, u\right), d\left(x_{n},\left[T x_{n}\right]_{\alpha}\right), d\left(u,[T u]_{\alpha}\right),\right. \\
\left.\frac{d\left(x_{n},[T u]_{\alpha}\right)+d\left(u,\left[T x_{n}\right]_{\alpha}\right)}{2}, \frac{d\left(x_{n},\left[T x_{n}\right]_{\alpha}\right) d\left(u,[T u]_{\alpha}\right)}{1+d\left(x_{n}, u\right)}\right\}+ \\
+k \min \left\{d\left(x_{n},\left[T x_{n}\right]_{\alpha}\right), d\left(u,\left[T x_{n}\right]_{\alpha}\right), \frac{d\left(x_{n},\left[T x_{n}\right]_{\alpha}\right) d\left(u,[T u]_{\alpha}\right)}{1+d\left(x_{n}, u\right)}\right\} \leq \\
\leq \alpha \max \left\{d\left(x_{n}, u\right), d\left(x_{n}, x_{n+1}\right), d\left(u,[T u]_{\alpha}\right),\right. \\
\left.\frac{d\left(x_{n},[T u]_{\alpha}\right)+d\left(u, x_{n+1}\right)}{2}, \frac{d\left(x_{n}, x_{n+1}\right) d\left(u,[T u]_{\alpha}\right)}{1+d\left(x_{n}, u\right)}\right\}+ \\
+\beta \min \left\{d\left(x_{n}, x_{n+1}\right), d\left(u, x_{n+1}\right), \frac{d\left(x_{n}, x_{n+1}\right) d\left(u,[T u]_{\alpha}\right)}{1+d\left(x_{n}, u\right)}\right\} .
\end{gathered}
$$

As $n \rightarrow \infty$ in (14), we have

$$
d\left(u,[T u]_{\alpha}\right) \leq \alpha \max \left\{d\left(u,[T u]_{\alpha}\right), \frac{d\left(u,[T u]_{\alpha}\right)}{2}, 0\right\} \leq \alpha d\left(u,[T u]_{\alpha}\right) .
$$

Since $1-\alpha>0$, (15) implies that $d\left(u,[T u]_{\alpha}\right)=0$, and, consequently, $u \in[T u]_{\alpha}$ for some $\alpha \in I_{-1}$.

Example 2. Let $X=\{1,2,3\},\{1\},\{2\},\{3\}$ be crisp sets. Define $d: X \times X \longrightarrow \mathbb{R}$ as follows:

$$
d(x, y)= \begin{cases}0, & \text { if } x=y \\ \frac{5}{18}, & \text { if } x \neq y \text { and } x, y \in X \backslash\{2\} \\ 1, & \text { if if } x \neq y \text { and } x, y \in X \backslash\{3\} \\ \frac{7}{18}, & \text { if if } x \neq y \text { and } x, y \in X \backslash\{1\}\end{cases}
$$

Define a fuzzy mapping $T: X \longrightarrow I^{X}$ as follows:

$$
\begin{gathered}
T(1)(t)=T(3)(t)= \begin{cases}\frac{7}{12}, & \text { if } t=1, \\
\frac{1}{3}, & \text { if } t=2, \\
0, & \text { if } t=3,\end{cases} \\
T(2)(t)= \begin{cases}0, & \text { if } t=1, \\
\frac{1}{5}, & \text { if } t=2 \\
\frac{7}{12}, & \text { if } t=3 .\end{cases}
\end{gathered}
$$


Then, for $\alpha=\frac{7}{12}$, we have

$$
[T x]_{\alpha}=\{t \in X: T(x)(t) \geq \alpha\}= \begin{cases}\{1\}, & \text { if } x \neq 2 \\ \{3\}, & \text { if } x=2 .\end{cases}
$$

Consider the following cases:

Case I: For $x \in X \backslash\{2\}$,

$$
d\left(x,[T x]_{\alpha}\right)=\inf \left\{d(x, y): y \in[T x]_{\alpha}\right\}=0 .
$$

Hence, for $x=y$ we have

$$
r(\alpha) d\left(x,[T x]_{\alpha}\right) \leq d(x, y) .
$$

Case II: For $x \in X \backslash\{1,3\}$,

$$
d\left(x,[T x]_{\alpha}\right)=\inf \left\{d(x, y): y \in[T x]_{\alpha}\right\}=\frac{7}{18} .
$$

Therefore, for $x \neq y$ we get

$$
r(\alpha) d\left(x,[T x]_{\alpha}\right)=\frac{7}{36} \leq d(x, y) .
$$

Thus, from the two cases, it follows that for any $\beta \geq 0$ there exists $\alpha=\frac{7}{12} \in I_{-1}$, such that

$$
r(\alpha) d\left(x,[T x]_{\alpha}\right) \leq d(x, y)
$$

implies

$$
H\left([T x]_{\alpha},[T y]_{\alpha}\right) \leq \alpha \bigvee(x, y)+\beta \bigwedge(x, y) .
$$

Consequently, all the hypotheses of Theorem 1 are satisfied to obtain $1 \in[T 1]_{\frac{7}{12}}$.

Next, we present a local fuzzy fixed point theorem for Suzuki-type $(\alpha, \beta)$-weak contractions. First, recall that an open ball with radius $r>0$, centered at $x_{0}$ in a metric space $X$, is given by

$$
B_{r}\left(x_{0}\right)=\left\{x \in X: d\left(x, x_{0}\right)<r\right\} .
$$

Theorem 2. Let $(X, d)$ be a complete metric space, $T: B_{r}\left(x_{0}\right) \rightarrow I^{X}$ be a Suzuki-type $(\alpha, \beta)$-weak contraction. Assume that for each $x \in X$ 
there exists $\alpha \in I_{-1}$ such that $[T x]_{\alpha}$ is a nonempty closed and bounded subset of $X$ and

$$
d\left(x_{0},\left[T x_{0}\right]_{\alpha}\right)<(1-\alpha) r
$$

Then $\operatorname{Fix}(T) \neq \emptyset$.

Proof. Let $0<\eta<r$ be such that $0<(1-\alpha)(1+\sqrt{\alpha}) \leq \frac{1}{1+\eta}, B_{\eta}^{*}\left(x_{0}\right) \subset B_{r}\left(x_{0}\right)$, and $d\left(x_{0},\left[T x_{0}\right]_{\alpha}\right)<(1-\alpha) \eta$. Then $(1-\alpha) \eta-d\left(x_{0},\left[T x_{0}\right]_{\alpha}\right)>0$. Choose $\gamma=(1-\alpha) \eta-d\left(x_{0},\left[T x_{0}\right]_{\alpha}\right)>0$; then there exists $x_{1} \in\left[T x_{0}\right]_{\alpha}$, such that $d\left(x_{0}, x_{1}\right)<d\left(x_{0},\left[T x_{0}\right]_{\alpha}\right)+\gamma$. Thus, $d\left(x_{0}, x_{1}\right)<(1-\alpha) \eta$. Now, for $\rho=\frac{1}{\sqrt{\alpha}}$ and $x_{1} \in\left[T x_{0}\right]_{\alpha}$, there exists $x_{2} \in\left[T x_{1}\right]_{\alpha}$, such that $d\left(x_{1}, x_{2}\right) \leq$ $\leq \rho H\left(\left[T x_{0}\right]_{\alpha},\left[T x_{1}\right]_{\alpha}\right)$. Note that

$$
r(\alpha) d\left(x_{0},\left[T x_{0}\right]_{\alpha}\right) \leq r(\alpha) d\left(x_{0}, x_{1}\right) \leq d\left(x_{0}, x_{1}\right),
$$

we have

$$
\begin{gathered}
d\left(x_{1}, x_{2}\right) \leq \rho H\left(\left[T x_{0}\right]_{\alpha},\left[T x_{1}\right]_{\alpha}\right)=\frac{1}{\sqrt{\alpha}} H\left(\left[T x_{0}\right]_{\alpha},\left[T x_{1}\right]_{\alpha}\right) \leq \\
\leq \sqrt{\alpha} \bigvee\left(x_{0}, x_{1}\right)+\frac{\beta}{\sqrt{\alpha}} \bigwedge\left(x_{0}, x_{1}\right) \leq \\
\leq \sqrt{\alpha} \max \left\{d\left(x_{0}, x_{1}\right), d\left(x_{0},\left[T x_{0}\right]_{\alpha}\right), d\left(x_{1},\left[T x_{1}\right]_{\alpha}\right),\right. \\
\left.\frac{d\left(x_{0},\left[T x_{1}\right]_{\alpha}\right)+d\left(x_{1},\left[T x_{0}\right]_{\alpha}\right)}{2}, \frac{d\left(x_{0},\left[T x_{0}\right]_{\alpha}\right) d\left(x_{1},\left[T x_{1}\right]_{\alpha}\right)}{1+d\left(x_{0}, x_{1}\right)}\right\}+ \\
+\frac{\beta}{\sqrt{\alpha}} \min \left\{d\left(x_{0},\left[T x_{0}\right]_{\alpha}\right), d\left(x_{1},\left[T x_{0}\right]_{\alpha}\right), \frac{d\left(x_{0},\left[T x_{0}\right]_{\alpha}\right) d\left(x_{1},\left[T x_{1}\right]_{\alpha}\right)}{1+d\left(x_{0}, x_{1}\right)}\right\} \leq \\
\leq \sqrt{\alpha} \max \left\{d\left(x_{0}, x_{1}\right), d\left(x_{0}, x_{1}\right), d\left(x_{1}, x_{2}\right),\right. \\
\left.\frac{d\left(x_{0}, x_{2}\right)+d\left(x_{1}, x_{1}\right)}{2}, \frac{d\left(x_{0}, x_{1}\right) d\left(x_{1}, x_{2}\right)}{1+d\left(x_{0}, x_{1}\right)}\right\}+ \\
+\frac{\beta}{\sqrt{\alpha}} \min \left\{d\left(x_{0}, x_{1}\right), d\left(x_{1}, x_{1}\right), \frac{d\left(x_{0}, x_{1}\right) d\left(x_{1}, x_{2}\right)}{1+d\left(x_{0}, x_{1}\right)}\right\} \leq \\
\leq \sqrt{\alpha} \max \left\{d\left(x_{0}, x_{1}\right), d\left(x_{1}, x_{2}\right), \frac{d\left(x_{0}, x_{1}\right)+d\left(x_{1}, x_{2}\right)}{2}\right\}+ \\
+\frac{\beta}{\sqrt{\alpha}} \min \left\{d\left(x_{0}, x_{1}\right), 0, d\left(x_{1}, x_{2}\right)\right\} \leq \sqrt{\alpha} \max \left\{d\left(x_{0}, x_{1}\right), d\left(x_{1}, x_{2}\right)\right\} . \quad(16)
\end{gathered}
$$


If $d\left(x_{0}, x_{1}\right) \leq d\left(x_{1}, x_{2}\right)$, then (16) gives

$$
d\left(x_{1}, x_{2}\right) \leq \sqrt{\alpha} d\left(x_{1}, x_{2}\right)<d\left(x_{1}, x_{2}\right):
$$

a contradiction. Hence, $d\left(x_{0}, x_{1}\right)>d\left(x_{1}, x_{2}\right)$ and

$$
d\left(x_{1}, x_{2}\right)<\sqrt{\alpha} d\left(x_{0}, x_{1}\right)<\sqrt{\alpha}(1-\alpha) \eta .
$$

Note that by the assumption and the triangle inequality, we have

$$
d\left(x_{0}, x_{2}\right) \leq d\left(x_{0}, x_{1}\right)+d\left(x_{1}, x_{2}\right)<(1-\alpha) \eta+\sqrt{\alpha}(1-\alpha) \eta \leq \frac{\eta}{1+\eta}<\eta .
$$

Hence, $x_{2} \in B_{\eta}\left(x_{0}\right)$. Continuing this process recursively, we generate a sequence $\left\{x_{n}\right\}_{n \in \mathbb{N}}$, such that

(a) $x_{n} \in B_{\eta}\left(x_{0}\right)$ for all $n \in \mathbb{N}$;

(b) $x_{n} \in\left[T x_{n}\right]_{\alpha}$ for each $n \in \mathbb{N}$;

(c) $d\left(x_{n}, x_{n+1}\right) \leq(\sqrt{\alpha})^{n}(1-\alpha) \eta$ for all $n \in \mathbb{N}$.

From $(c)$, it follows, by usual arguments, that $\left\{x_{n}\right\}_{n \in \mathbb{N}}$ is a Cauchy sequence and converges to some $u \in B_{r}\left(x_{0}\right)$. From here, following the steps in the proof of Theorem 1, we conclude that $\operatorname{Fix}(T) \neq \emptyset$.

Corollary 1. Let $(X, d)$ be a complete metric space and $T: X \rightarrow C B(X)$ be a multi-valued mapping. Assume that there exists an $\alpha \in I_{-1}$ and some constants $\beta \geq 0$, such that for all $x, y \in X$,

$$
r(\alpha) d(x, T x) \leq d(x, y)
$$

implies

$$
H(T x, T y) \leq \alpha \bigvee(x, y)+\beta \bigwedge(x, y),
$$

where the mapping $r: I_{-1} \rightarrow I^{+1}$ is defined by

$$
r(\alpha)= \begin{cases}1, & \text { if } 0 \leq \alpha<\frac{1}{2} \\ 1-\alpha, & \text { if } \frac{1}{2} \leq \alpha<1\end{cases}
$$

Then $\operatorname{Fix}(T) \neq \emptyset$.

Proof. Let $I_{-1}=(0,1)$ and $\alpha \in I_{-1}$ be arbitrary. Consider a mapping $\varpi: X \rightarrow I_{-1}$ and a fuzzy mapping $\digamma: X \rightarrow I^{X}$ defined by

$$
\digamma(x)(t)= \begin{cases}\varpi(x), & \text { if } t \in T x \\ 0, & \text { if } t \notin T x .\end{cases}
$$


Then

$$
[\digamma x]_{\alpha}=\{t \in X: \digamma(x)(t) \geq \varpi(x)\}=T x .
$$

Therefore,

$$
d(x, y) \geq r(\alpha) d(x, T x)=r(\alpha) d\left(x,[\digamma x]_{\alpha}\right) .
$$

Thus, Theorem 1 can be applied to finding $u \in X$, such that $u \in T u$. $\square$

In the following, we provide an example to highlight the generality of Corollary 1.

Example 3. Let $X=\{a, b, c, p, q\}$ and $d: X \times X \rightarrow[0, \infty)$ be defined by

$$
\begin{gathered}
d(a, b)=d(a, c)=9, \\
d(b, q)=d(c, p)=d(c, q)=d(b, c)=14, \\
d(a, p)=d(a, q)=18, \\
d(b, p)=16, d(p, q)=10, \\
d(u, u)=0 \text { and } d(u, v)=d(v, u) \text { for all } u, v \in X .
\end{gathered}
$$

Define $T: X \rightarrow C B(X)$ by

$$
T u= \begin{cases}\{c\}, & \text { if } u \in X \backslash\{a, b, c, p\} \\ \{a, b\}, & \text { if } u \in X \backslash\{a, b, c, q\} \\ \{a\}, & \text { if } u \in X \backslash\{p, q\} .\end{cases}
$$

Now, it is easy to verify that all the assumptions of Corollary 1 are satisfied with $\alpha=\frac{8}{13}$ and $\beta=6$. In fact,

$$
r(\alpha) d(p, T p)=9.85 \leq d(p, q)
$$

implies

$$
\begin{aligned}
H(T p, T q)=H & (\{a, b\},\{c\})=14 \leq \alpha \bigvee(p, q)+\beta \bigwedge(p, q) \leq \\
& \leq \frac{8}{13} \max \{10,14,14,14,18\}+6 \min \{14,14,18\} \leq 95
\end{aligned}
$$

Further, we see that there exists $u=a \in X$, such that $u \in T u$. On the other hand, observe that if we set $u=p$ and $v=q$, then 


$$
\begin{aligned}
& \bigvee(p, q)=\max \{d(p, q), d(p, T p), d(q, T q) \\
& \left.\frac{d(p, T q)+d(q, T p)}{2}, \frac{d(p, T p) d(q, T q)}{1+d(p, q)}\right\}= \\
& \quad=\max \{10,14,14,14,18\}=18
\end{aligned}
$$

and

$$
H(T p, T q)=14>11.08=\alpha \bigvee(p, q) .
$$

Therefore, the main results in $[8,9]$ cannot be applied in this case to find a fixed point of $T$.

5. Application in Homotopy. In this section, we apply Theorem 2 to prove a homotopy result. First, for convenience, we recall the following familiar definitions.

Definition 2. A relation $\leq$ is a total order on a set $U$ if for all $s, t, u \in U$, the following conditions hold:

(i) Reflexivity: $s \leq s$;

(ii) Antisymmetry: if $s \leq t$ and $t \leq s$, then $s=t$;

(iii) Transitivity: if $s \leq t$ and $t \leq u$, then $s \leq u$;

(iv) Comparability: for every $s, t \in U$, either $s \leq t$ or $t \leq s$.

Recall that if the set $U$ satisfies only the axioms $(i)-(i i i)$, then it is said to be partially ordered. In what follows, we shall call a totally ordered set a chain.

Lemma 1. (Kuratowski-Zorn's Lemma ) If $U$ is any nonempty partially ordered set in which every chain has an upper bound, $U$ has a maximal element.

Definition 3. Let $X_{1}$ and $X_{2}$ be any two topological spaces and $\pi, \omega$ : $X_{1} \rightarrow X_{2}$ be continuous functions. A function $H: X_{1} \times[0,1] \rightarrow X_{2}$, such that for any $u \in X_{1} H(u, 0)=\pi(u)$ and $H(u, 1)=\omega(u)$, is called a homotopy between $\pi$ and $\omega$.

We shall denote the boundary of a set $U$ by $B d(U)$.

Theorem 3. Let $(X, d)$ be a complete metric space and $U$ be an open subset of $X$. If $M: \bar{U} \times[0,1] \rightarrow I^{X}$ satisfies the following conditions:

(h1) $u \notin M(u, t)$ for every $u \in B d(U)$ and $t \in[0,1]$; 
(h2) $M(\cdot, t): \bar{U} \longrightarrow I^{X}$ is a Suzuki-type $(\alpha, \beta)$-weak contraction for all $t \in[0,1]$

(h3) there exist a nondecreasing function $f:[0,1] \rightarrow \mathbb{R}$, such that $H(M(u, t), M(u, s)) \leq|f(t)-f(s)|$ for all $s, t \in[0,1]$ and each $u \in \bar{U}$;

(h4) $M: \bar{U} \times[0,1] \rightarrow I^{X}$ is closed and bounded;

then $M(\cdot, 0)$ has a fixed point.

Proof. Assume that $p$ is a fixed point of $M(\cdot, 0)$. Then, by condition (h1), $p \in U$. Consider the set $\Lambda^{*}$, given by

$$
\bigwedge^{*}=\{(t, u) \in[0,1] \times U: u \in M(u, t)\} .
$$

Note that $(0, p) \in \bigwedge^{*}$; hence $\bigwedge^{*} \neq \emptyset$. Let $\alpha \in I_{-1}=(0,1)$ and define a partial order $\leq$ on $\bigwedge^{*}$ as follows:

$$
(t, u) \leq(s, v) \text { if and only if } t \leq s \text { and } d(u, v) \leq \frac{2}{1-\alpha}[f(s)-f(t)] .
$$

Suppose $\Omega$ is a chain of $\bigwedge^{*}$ and $t^{*}:=\sup \{t:(t, u) \in \Omega\}$. Assume that $\left\{t_{n}, u_{n}\right\}$ is a sequence in $\Omega$, such that $\left(t_{n}, u_{n}\right) \leq\left(t_{n+1}, u_{n+1}\right)$ and $t_{n} \rightarrow t^{*}$ as $n \rightarrow \infty$. Then, for all positive integers $m, n(m>n)$,

$$
d\left(u_{m}, u_{n}\right) \leq \frac{2}{1-\alpha}\left|f\left(t_{m}\right)-f\left(t_{n}\right)\right| .
$$

As $m, n \rightarrow \infty$ in (17), we get $d\left(u_{m}, u_{n}\right) \rightarrow 0$. Hence, $\left\{u_{n}\right\}_{n \in \mathbb{N}}$ is a Cauchy sequence and converges to some $u^{*} \in X$. Since $M$ is closed and $u_{n} \in M\left(u_{n}, t_{n}\right)$, therefore, $u^{*} \in M\left(u^{*}, t^{*}\right)$. From condition (h1), $u^{*} \in U$. Thus, $\left(t^{*}, u^{*}\right) \in \Lambda^{*}$. Since $\Omega$ is a chain, hence $(t, u) \leq\left(t^{*}, u^{*}\right)$ for all $(t, u) \in \Omega$. In other words, $\left(t^{*}, u^{*}\right)$ is an upper bound of $\Omega$. Thus, by Kuratowski-Zorn Lemma, $\Lambda^{*}$ has a maximal element $\left(t_{0}, u_{0}\right)$. Next, we show that $t_{0}=1$. Suppose on the contrary: that $t_{0}<1$. Let $r=\frac{2}{1-\alpha}\left|f(t)-f\left(t_{0}\right)\right|>0$ with $t \in\left(t_{0}, 1\right]$, such that $B_{r}\left(u_{0}\right) \subset U$. Note that by condition (h3)

$$
\begin{aligned}
d\left(u_{0}, M\left(u_{0}, t\right)\right) \leq d\left(u_{0}, M\left(u_{0}, t_{0}\right)\right)+H\left(M\left(u_{0}, t_{0}\right), M\left(u_{0}, t\right)\right) & \leq \\
& \leq\left|f(t)-f\left(t_{0}\right)\right|=\frac{(1-\alpha) r}{2}<(1-\alpha) r .
\end{aligned}
$$


Therefore, $M(\cdot, t): B_{r}\left(u_{0}\right) \rightarrow I^{X}$ satisfies all the assumptions of Theorem 2 for every $t \in[0,1]$. Consequently, there exists $u \in B_{r}\left(u_{0}\right)$, such that $u \in M(u, t)$, which implies that $(t, u) \in \bigwedge^{*}$ for all $t \in[0,1]$. Now,

$$
d\left(u_{0}, u\right) \leq r=\frac{2}{1-\alpha}\left|f(t)-f\left(t_{0}\right)\right|,
$$

yields $\left(t_{0}, u_{0}\right)<(t, u)$, which is a contradiction to the fact that $\left(t_{0}, u_{0}\right)$ is maximal. Conversely, assume that $M(\cdot, 1)$ has a fixed point; then, using similar steps as above, one can show that $M(\cdot, 0)$ has a fixed point.

Competing Interests. The authors declare that they have no competing interests.

Acknowledgments. The authors thank the editors and the anonymous reviewers for their valuable suggestions and comments, which greatly helped us in improving this paper.

\section{References}

[1] Azam, A., Arshad, M., and Vetro, P. On a pair of fuzzy $\varphi$-contractive mappings. Mathematical and Computer Modelling, 2010, vol. 52, no. 1-2, pp. 207-214.

[2] Azam, A., and Beg, I. Common fixed points of fuzzy maps. Mathematical and computer modelling, 2009, vol. 49, no. 7, pp. 1331-1336.

[3] Azam, A., Arshad, M., and Beg, I. Fixed points of fuzzy contractive and fuzzy locally contractive maps. Chaos, Solitons and Fractals, 2009, vol. 42, no. 5, pp. 2836-2841.

[4] Banach, S. Sur les operations dans les ensembles abstraits et leur application aux equations integrales. Fund. math, 1922, vol. 3, no. 1, pp. 133-181.

[5] Berinde, V. Approximating fixed points of weak contractions using the Picard iteration. In Nonlinear Analysis Forum, 2004, vol. 9, pp. 43-54.

[6] Butnariu, D. Fixed points for fuzzy mappings. Fuzzy sets and Systems, 1982, vol. 7, no. 2, pp. 191-207.

[7] Chatterjea, S.K. Fixed point theorems. C.R. Acad. Bulgare Sci., 1972, vol. 25 , pp. $727-730$.

[8] Ciric, L. B. Fixed points for generalized multi-valued contractions. Math. Vesnik, 1972, vol. 9, no. 24, pp. 265-272.

[9] Doric, D., Lazovic, R. Some Suzuki-type fixed point theorems for generalized multivalued mappings and applications. Fixed Point Theory and Applications, 2011, vol. 40, no. 1 .

DOI: https://doi.org/10.1186/1687-1812-2011-40. 
[10] Edelstein, M. On fixed and periodic points under contractive mappings. Journal of the London Mathematical Society, 1962, vol. 1, no. 1, pp. 74-79.

[11] Heilpern, S. Fuzzy mappings and fixed point theorem. Journal of Mathematical Analysis and Applications, 1981, vol. 83, no. 2, pp. 566-569.

[12] Humaira, M. S., and Tunc, C. Fuzzy fixed point results via rational type contractions involving control functions in complex-valued metric spaces. Appl. Math, 2018, vol. 12, no. 4, pp. 861-875.

[13] Kannan, R. Some results on fixed points II. The American Mathematical Monthly, 1969, vol. 76, no. 4, pp. 405-408.

[14] Rome, B. E., Sarwar, M., and Kumam, P. Fixed point theorems via $\alpha-\varrho-$ fuzzy contraction. Axioms, 2019, 8(2), 69.

DOI: https://doi.org/10.3390/axioms8020069.

[15] Mohammed, S. S. On bilateral fuzzy contractions. Functional Analysis, Approximation and Computation, 2020, vol. 12, no. 1, pp. 1-13.

[16] Sarwar, M., and Kishore, G. N. V. Fuzzy fixed point results for contractive mapping with applications. Complexity, 2018.

[17] Sarwar, M., and Kumam, P. Common fixed point results for fuzzy mappings on complex-valued metric spaces with homotopy results. Symmetry, 2019, 11(1), 61. DOI: https://doi.org/10.3390/sym11010061.

[18] Sarwar, M., and Li, T. Fuzzy fixed point results and applications to ordinary fuzzy differential equations in complex valued metric spaces. Hacettepe Journal of Mathematics and Statistics, 2019, vol. 48, no. 6, pp. 1712-1728.

[19] Suzuki, T. A generalized Banach contraction principle that characterizes metric completeness. Proceedings of the American Mathematical Society, 2008, vol. 136, no. 5, pp. 1861-1869.

[20] Weiss, M. D. Fixed points, separation, and induced topologies for fuzzy sets. Journal of Mathematical Analysis and Applications, 1975, vol. 50, no. 1, pp. $142-150$.

[21] Zadeh, L. A. Fuzzy sets. Information and control, 1965, vol. 8(3), pp. 338-353.

[22] Zamfirescu, T. Fixed point theorems in metric spaces. Archiv der Mathematik, 1972, vol. 23(1), pp. 292-298.

Received July 19, 2019.

In revised form, February 16, 2020.

Accepted February 19, 2020.

Published online April 14, 2020. 
Mohammed Shehu Shagari

Department of Mathematics, COMSATS University

Chak Shahzad, Islamabad, 44000, Pakistan;

Department of Mathematics, Faculty of Physical Sciences

Ahmadu Bello University, Nigeria

E-mail: ssmohammed@abu.edu.ng

Akbar Azam

Department of Mathematics, COMSATS University

Chak Shahzad, Islamabad, 44000, Pakistan

E-mail: akbarazam@yahoo.com 